\title{
Publisher's Note: Dynamical realization of magnetic states in a strongly interacting Bose mixture [Phys. Rev. A 95, 023612 (2017)]
}

\author{
R. E. Barfknecht, A. Foerster, and N. T. Zinner
}

(Received 2 May 2017; published 10 May 2017)

DOI: 10.1103/PhysRevA.95.059903

This paper was published online on 14 February 2017 with incorrect placement of panels (c) and (d) in Fig. 6. The panels have been transposed as of 1 May 2017. The panels are incorrect in the printed version of the journal; therefore for the benefit of the print readership, the figure has been replicated below.

(a)
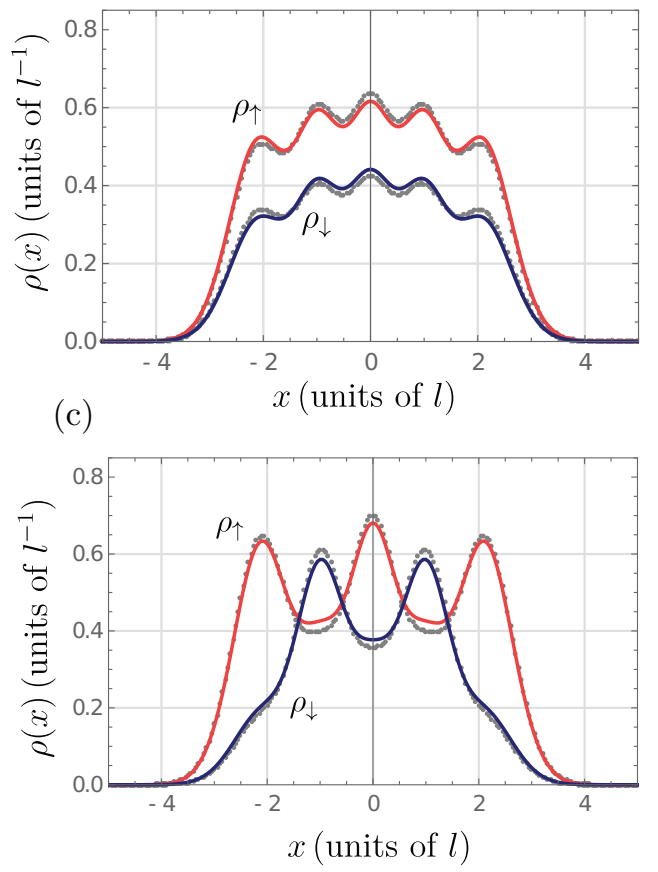

(b)

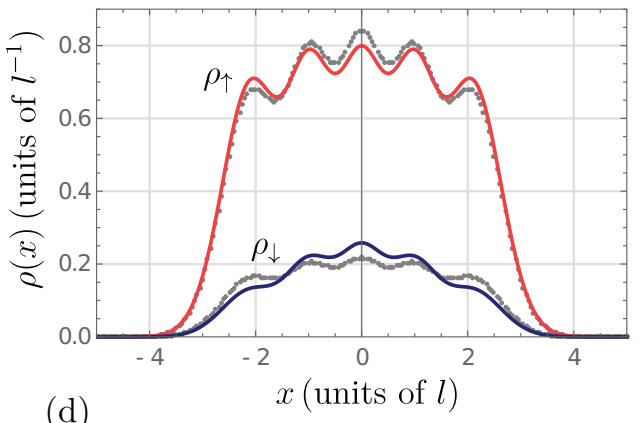

(d)

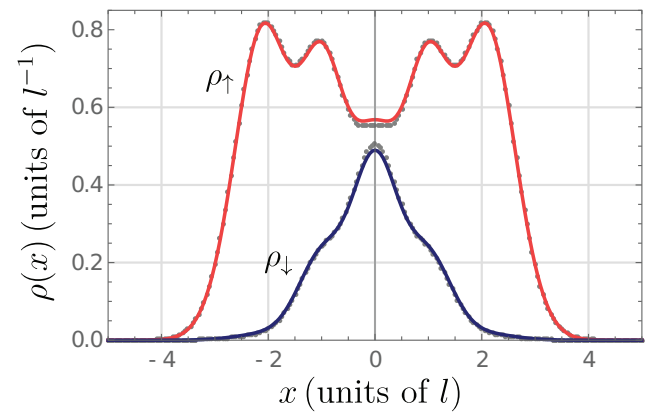

FIG. 6. Intermediate and final profiles for the time evolution of spin densities. Light (red) and dark (blue) curves indicate the $\uparrow$ and $\downarrow$ components, respectively. Upper panels show the profiles at $\kappa=1.1\left(t=0.5 \times 10^{4}[\tau]\right)$ for the (a) $N_{\uparrow}=3, N_{\downarrow}=2$ and (b) $N_{\uparrow}=4, N_{\downarrow}=1$ cases. The gray dots correspond to the results obtained with $g=-100$ and $\kappa \rightarrow \infty$. The lower panels show the final profiles $\left(t=0.5 \times 10^{5}[\tau]\right.$, $\kappa \sim 10$ ) for the (c) $N_{\uparrow}=3, N_{\downarrow}=2$ and (d) $N_{\uparrow}=4, N_{\downarrow}=1$ cases, now compared to the limiting case of $g=100$ and $\kappa \rightarrow \infty$ (gray dots). 